\title{
Famílias de crianças com síndrome de Down: sentimentos, modos de vida e estresse parental ${ }^{1}$
}

\author{
Nara Liana Pereira-Silva \\ Maria Auxiliadora Dessen \\ Universidade de Brasília
}

\begin{abstract}
RESUMO
Considerando a importância de se conhecer os processos familiares para a compreensão do desenvolvimento de crianças que apresentam deficiência, este estudo comparativo descreve aspectos do funcionamento familiar e do desenvolvimento de crianças com e sem síndrome de Down, destacando o modo de vida das famílias, o estresse parental, bem como as reações e os sentimentos dos genitores frente ao diagnóstico do filho com síndrome de Down. Participaram deste estudo dez famílias compostas por mãe, pai e filhos morando juntos; cinco delas com uma criança com síndrome de Down. As famílias foram visitadas ao longo de dois anos, em intervalos de seis meses, sendo administrados questionários, entrevistas semi-estruturadas e uma escala de estresse parental a pais e mães. Os resultados mostraram que a rotina das famílias foi similar e que mães de crianças com síndrome de Down apresentaram índices mais elevados de estresse que mães de crianças com desenvolvimento típico. Os dados sugerem similaridades nos modos de vida das famílias e diferenças no estresse parental, que precisam ser exploradas mais detalhadamente.
\end{abstract}

Palavras-chave: síndrome de Down; famílias; crianças pré-escolares; estresse parental

\section{ABSTRACT \\ Families of children with Down syndrome: feelings, lifestyle and parental stress}

Considering the importance of investigating family processes to understand the development of disabled children, this comparative study describes some developmental aspects of children with and without Down syndrome and their family functioning. It focuses on family lifestyles, parental stress and parental reactions and feelings when facing diagnosis of Down syndrome in their children. Ten families participated in this study, all consisting of mother, father and children leaving together; five of them having a child with Down syndrome. These families were visited at a six-month interval during a two-year period, when questionnaires, semi-structured interviews and a parental stress scale were administered to each parent. The results show that the routines of both families are similar and that the mothers of Down syndrome children are more stressful than those of typically developing children. The empirical data suggests lifestyle similarities and parental stress differences among those family groups, suggesting the need of further investigation.

Keywords: Down syndrome; families; preschoolers; parental stress

Há algumas décadas, o panorama apresentado pelas pesquisas na área de deficiência mental (DM) e família era desanimador, ressaltando, sobretudo, os aspectos patológicos em detrimento dos aspectos saudáveis, tanto do desenvolvimento da criança quanto do funcionamento familair. Contudo, nos últimos anos, com a ampliação do número de pesquisas nesta área, tem-se reconhecido a possibilidade de uma adaptação mais positiva da família a este novo membro (Pereira-Silva \& Dessen, 2003, 2004; Shapiro, Blacher \& Lopez, 1998;). Neste processo, o envol- vimento entre a família e a criança é de extrema importância, pois o modo como a criança é incluída no grupo afeta diretamente o seu desenvolvimento (Bronfenbrenner, 1979/1996, 1999).

Não há dúvidas de que o nascimento de uma criança DM tem um impacto profundo e significativo para toda a família (Casarin, 1999; Krauss \& Seltzer, 1998; Minnes, 1998; Pereira-Silva \& Dessen, 2002; Regen, Ardore \& Hoffmann, 1993; Saad, 2003). Entretanto, a literatura tem focalizado sua atenção quase que exclusivamente às mães, apontando diferenças marcantes 
entre mães de crianças com e sem DM e, também, entre mães e pais de crianças com deficiência mental (Dyson, 1997; Hornby, 1995; Lamb \& Billings, 1997; Shapiro \& cols., 1998; Slonims \& McChonachie, 2006). Essas evidências indicam que as mães continuam sendo mais fortemente afetadas pelas obrigações de cuidados com a criança DM. Apesar disso, não se tem clareza de que os altos níveis de sintomatologia emocional e física dessas mães estejam relacionados à presença da criança ou à rede de variáveis mediadoras ou, ainda, às diferenças culturais entre homens e mulheres (Shapiro \& cols., 1998).

Uma das sintomatologias freqüentemente investigadas na área de deficiência mental e família é o estresse. Há dados que mostram níveis similares de estresse em mães e pais de crianças com DM em idade escolar (Dyson, 1997). Mas, a maioria dos estudos aponta um nível mais elevado de estresse em mães do que em pais de crianças com DM, ou mesmo entre elas e mães de crianças com desenvolvimento típico ${ }^{2}$ (Dyson, 1997; Hornby, 1995; Lamb \& Billings, 1997; Sloper, Knussen, Turner \& Cunningham, 1991). Os dados de Shapiro e cols. (1998) também indicam a existência de um estresse diferencial quando se comparam famílias de crianças com e sem DM. Segundo estes autores, o estresse depende, em parte, das características da criança com deficiência, isto é, dos diferentes tipos de deficiências e de suas combinações e do impacto da sobrecarga de cuidado com a criança.

A sobrecarga adicional de cuidados da família com a criança DM está presente durante todo o curso de vida familiar, estando, muitas vezes, associada aos sentimentos de ansiedade e incerteza, sobretudo em relação às questões de sobrevivência da criança e de seu desenvolvimento, da necessidade de cuidados prolongados e do próprio impacto desse cuidado sobre a vida pessoal da mãe (Shapiro \& cols., 1998). Contudo, a sobrecarga não recai apenas sobre a mãe. O estudo de Rodrigue, Morgan e Geffken (1992) evidencia que pais de crianças com síndrome de Down experienciam mais sobrecarga nas suas vidas diárias que pais de crianças com desenvolvimento típico. A sobrecarga, portanto, vem sendo tratada como um fator desencadeante do estresse e de sentimentos vivenciados pelos genitores de crianças com deficiência mental.

Além da sobrecarga, a depressão e o ajustamento psicológico também são aspectos importantes do funcionamento individual de pais e mães de crianças com deficiência mental, uma vez que seus efeitos têm re- percussões na dinâmica do grupo familiar, podendo alterar as relações entre seus membros. Os estudos mostram que: (a) mães de crianças DM apresentam taxas mais altas de depressão e enfrentam mais problemas com o ajustamento psicológico que os pais de crianças deste grupo (Glidden \& Floyd, 1997; Hastings, Daley \& Burns, 2006; Negrin \& Cristante, 1996; Singer, 2006); (b) mães de crianças com desenvolvimento típico são mais ajustadas e apresentam taxas mais baixas de depressão que mães de crianças DM (Carr, 1988; Shapiro \& cols., 1998).

A literatura tem apontado também que genitores de crianças DM apresentam mais instabilidade emocional que os de crianças com desenvolvimento típico (Ali \& cols., 1994; Hastings \& cols., 2006; Sloper \& cols., 1991). A mágoa, o sofrimento, um contínuo processo de luto (Leary \& Verth, 1995) e a culpa (Ali \& cols., 1994) são sentimentos relatados pelos genitores como conseqüência de se ter um filho com deficiência mental. Entretanto, os genitores, sobretudo aqueles de crianças com síndrome de Down, relatam apreço e preocupação com sua criança (Pereira-Silva \& Dessen, 2004). Os estudos têm, portanto, focalizado mais os aspectos patológicos do funcionamento de famílias de crianças DM do que suas possibilidades adaptativas e saudáveis.

As expectativas dos genitores quanto ao desenvolvimento e a vida futura dos filhos também afetam a dinâmica das relações familiares (Pereira-Silva \& Dessen, 2001, 2003). Com relação às crianças com DM, estas expectativas parecem depender do gênero da criança, do tipo de deficiência e sua gravidade, dentre outros fatores. Isto é evidenciado, por exemplo, no estudo de Minnes (1998), em que as respostas dos genitores quanto às expectativas em relação ao filho foram diferentes e dependentes do sexo da criança com deficiência. Por outro lado, em um estudo conduzido no Brasil, observou-se que as expectativas das mães estavam mais associadas à preocupação com o desenvolvimento geral de sua criança do que com outros fatores (Brunhara \& Petean, 1999). Contudo, por haver problemas metodológicos em muitos estudos, Minnes (1998) sugere uma reavaliação dos dados disponíveis na literatura a respeito das expectativas dos genitores em relação à sua criança com DM.

Em síntese, o desenvolvimento de crianças com deficiência e a dinâmica familiar estão intimamente relacionados, influenciando-se mutuamente. Isto significa que a pesquisa deve levar em conta ambos os 
aspectos e, também, as inter-relações com os demais fatores que interferem, direta e indiretamente, na dinâmica do funcionamento das famílias de crianças com DM, incluindo aquelas com síndrome de Down. Pouco se conhece sobre o funcionamento destas famílias, especialmente no Brasil (Dessen \& Pereira-Silva, 2000). Assim, o presente estudo tem como objetivo descrever o funcionamento de famílias de crianças com síndrome de Down, usando como recurso metodológico a comparação com famílias de crianças com desenvolvimento típico. Ênfase é dada (a) à descrição dos aspectos do desenvolvimento das crianças, (b) ao modo de vida das famílias, (c) ao estresse parental e (d) aos sentimentos e reações dos genitores ao receberem a notícia do diagnóstico da criança com síndrome de Down.

\section{MÉTODO}

\section{Participantes}

Participaram deste estudo dez famílias compostas por mãe, pai e uma criança-alvo. Em cinco famílias, a criança-alvo possuía síndrome de Down (SD) e nas outras cinco, a criança-alvo não apresentava qualquer deficiência, isto é, tinha desenvolvimento típico (DT), de acordo com os relatos dos genitores e de suas professoras. Todos os membros da família moravam juntos em cidades satélites de Brasília $(n=9)$ ou no Plano Piloto $(n=1)$. Quatro crianças eram do sexo masculino e seis do sexo feminino, com idades que variavam entre dois e quatro anos, por ocasião da coleta de dados. Duas crianças não possuíam irmãos, enquanto as demais possuíam de um a cinco irmãos.

Nas famílias de crianças com síndrome de Down, a idade média das mães era de 37 anos e a dos pais de 33 anos; já nas famílias de crianças com desenvolvimento típico, a média de idade das mães era de 33 anos e a dos pais de 35 anos. Os pais das crianças com síndrome de Down exerciam as seguintes ocupações: radialista, administrador (aposentado), autônomo, militar e ajudante de serviços gerais. Já os pais das crianças com desenvolvimento típico exerciam funções de vendedor (dois pais), funcionário público, militar e auxiliar de escritório. Quanto às mães das crianças com síndrome de Down, duas não trabalhavam fora de casa, uma era professora e duas eram funcionárias públicas. Das cinco mães de crianças com desenvolvimento típico, três não trabalhavam fora de casa, uma era auxiliar de limpeza e a outra era funcionária pública. A renda familiar média era de 7,2 salários mínimos.
Com relação à escolaridade das mães das crianças com síndrome de Down, duas possuíam o ensino fundamental incompleto, uma o ensino médio completo e duas completaram o curso superior. Quanto aos pais destas crianças, dois tinham o ensino fundamental incompleto, um o ensino fundamental completo, dois completaram o curso superior e um ainda o estava cursando. No que se refere às mães das crianças com desenvolvimento típico, três tinham o ensino médio, uma completou o ensino superior e a outra não tinha completado o ensino fundamental. Já dos pais, um possuía o ensino fundamental completo e um incompleto, dois tinham o ensino superior completo e um ainda cursava o ensino superior.

\section{Procedimentos}

A coleta de dados com as dez famílias foi efetuada na própria residência das mesmas, ao longo de dois anos, com intervalos de seis meses entre as visitas. Foram utilizados os seguintes instrumentos: (a) questionário de caracterização da família, (b) entrevistas semi-estruturadas e (c) escala de estresse parental. Na primeira visita, por questões éticas, foi assinado o termo de consentimento para participação na pesquisa e, logo em seguida, o questionário de caracterização da família foi aplicado ao pai ou à mãe, com a finalidade de obter informações sociais e demográficas e da estrutura familiar, das atividades rotineiras e de lazer da família.

As entrevistas foram realizadas com o pai e a mãe, totalizando 30 entrevistas, todas elas registradas em um gravador da marca Panasonic. A entrevista inicial consistia de questões sobre a história de vida da criança, o diagnóstico, os hábitos e os costumes da criança e da família, as características comportamentais e emocionais da criança e as crenças, valores e expectativas dos genitores em relação ao filho/filha. Para as famílias de crianças com desenvolvimento típico, foram suprimidas as questões relativas ao diagnóstico e todas aquelas específicas às crianças com síndrome de Down. Esta entrevista foi realizada com a mãe e com o pai, separadamente $(n=20)$. A entrevista final com os genitores focalizou as mudanças ocorridas com a criança e com a família durante o período de realização da coleta de dados, e foi administrada à mãe e ao pai, conjuntamente $(n=10)$.

Com a finalidade de verificar o índice de estresse dos genitores decorrente de suas relações com o filho com síndrome de Down e com a criança com desenvolvimento típico selecionada como foco do estudo, 
aplicou-se uma escala de estresse parental denominada de Índice de Estresse Parental - PSI (Abidin, 1995). Esta escala destina-se a medir o estresse percebido pelos genitores por meio de três sub-escalas. A primeira sub-escala mede o sofrimento e a angústia vivenciados pelo genitor em seu papel de pai/mãe. A sub-escala 'interações disfuncionais entre genitorcriança' avalia as percepções que os genitores têm de seus filhos que não são compatíveis com as suas expectativas, bem como as percepções de suas interações com sua criança que não reforçam o seu papel de pai/mãe. A sub-escala 'criança difícil' focaliza algumas características comportamentais básicas de crianças que as tornam fáceis ou difíceis de manejar.

As entrevistas foram transcritas na íntegra, seguindo a sequiência do roteiro preestabelecido, com o objetivo de identificar os aspectos relevantes em cada questão. Em seguida, foram selecionadas e definidas as categorias que melhor agrupassem os conteúdos identificados. Os dados transcritos foram, então, tabulados e as frequiências das categorias computadas. O índice de estresse parental foi calculado somando-se as respostas de pais e mães, separadamente, obtendose o escore bruto indicativo do nível de estresse experienciado por eles. $\mathrm{O}$ escore bruto mínimo, na população canadense, é de 94 e o máximo é de 117 (Bigras, Venet \& Normandeau, 2002). Os resultados brutos foram comparados com os de uma amostra de mães canadenses (Bigras \& cols., 2002), considerando não haver validação deste instrumento para a população de pais (M. Bigras, comunicação pessoal, 05.02.2002).

\section{RESULTADOS}

Os resultados focalizam, primeiramente, as características comportamentais e os aspectos de desenvolvimento diferenciadores entre crianças com SD e com DT. Em seguida, é descrito o modo de vida das famílias, focalizando as mudanças ocorridas no sistema familiar e com as crianças, ao longo de dois anos. Posteriormente, o nível de estresse parental vivenciado pelos genitores é apresentado, para ambos os tipos de família, bem como as reações e os sentimentos das famílias de crianças com síndrome de Down diante do diagnóstico.

\section{Desenvolvimento das crianças com e sem Síndrome de Down}

Há diferenças no desenvolvimento das crianças SD e DT? De acordo com os genitores, as crianças SD foram percebidas como tendo um desenvolvimento motor e de linguagem mais lento que as crianças DT, havendo uma diferença de 3 a 5 meses no que se refere ao sentar, de até 11 meses no engatinhar e de até 21 meses no andar. Em geral, as crianças SD e DT sustentaram a cabeça em idades similares. Com relação ao desenvolvimento da linguagem, todas as crianças SD começaram a falar após os dois anos de idade, enquanto as crianças DT iniciaram entre 9 meses e um ano de idade, excetuando-se uma criança DT, que começou a falar em uma idade semelhante à idade das crianças SD. A Tabela 1 especifica as idades cronológicas em que as crianças sustentaram a cabeça, sentaram, engatinharam, andaram e falaram, segundo o relato das mães.

Tabela 1. Comparação de aspectos do desenvolvimento motor entre pares de crianças, segundo os relatos das mães

\begin{tabular}{|c|c|c|c|c|c|c|c|c|c|c|}
\hline \multirow{2}{*}{ 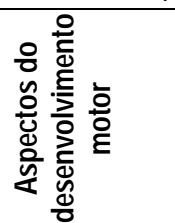 } & \multicolumn{10}{|c|}{$\begin{array}{c}\text { Crianças } \\
\text { Idade cronológica }\end{array}$} \\
\hline & $\begin{array}{c}\text { SD1 } \\
\text { (2 anos) }\end{array}$ & $\begin{array}{c}\text { DT1 } \\
(2 \mathrm{a} .5 \mathrm{~m})\end{array}$ & $\begin{array}{c}\text { SD2 } \\
\text { (2 anos) }\end{array}$ & $\begin{array}{c}\text { DT2 } \\
(3 a .10 \mathrm{~m})\end{array}$ & $\begin{array}{c}\text { SD3 } \\
(2 a .7 m)\end{array}$ & $\begin{array}{c}\text { DT3 } \\
(3 a .1 \mathrm{~m})\end{array}$ & $\begin{array}{c}\text { SD4 } \\
(2 \mathrm{a} .7 \mathrm{~m})\end{array}$ & $\begin{array}{c}\text { DT4 } \\
(4 \mathrm{a} .2 \mathrm{~m})\end{array}$ & $\begin{array}{c}\text { SD5 } \\
(3 a .3 \mathrm{~m})\end{array}$ & $\begin{array}{c}\text { DT5 } \\
(3 a .4 m)\end{array}$ \\
\hline $\begin{array}{ll}\begin{array}{l}\text { Sustentou } \\
\text { cabeça }\end{array} & \text { a }\end{array}$ & $8 m$ & $3 \mathrm{~m}$ & --- & 1 mês & --- & $2 m$ & $3 m$ & $4 \mathrm{~m}$ & $3 \mathrm{~m}$ & $1 \mathrm{~m}$ \\
\hline Sentou & $10 \mathrm{~m}$ & $7 \mathrm{~m}$ & $10 \mathrm{~m}$ & $6 \mathrm{~m}$ & $8 m$ & $5 \mathrm{~m}$ & $10 \mathrm{~m}$ & $6 \mathrm{~m}$ & $7 \mathrm{~m}$ & $6 \mathrm{~m}$ \\
\hline Engatinhou & $1 a .2 m$ & $7 \mathrm{~m}$ & 1 ano & $7 \mathrm{~m}$ & $8 \mathrm{~m}$ & $9 \mathrm{~m}$ & 1 ano & $7 \mathrm{~m}$ & $1 a .6 m$ & $7 \mathrm{~m}$ \\
\hline Andou & $2 a .6 m$ & $1 a .6 m$ & $2 a .2 m$ & $1 \mathrm{a} .1 \mathrm{~m}$ & $2 a .6 m$ & 1 ano & $1 a .10 \mathrm{~m}$ & $10 \mathrm{~m}$ & $1 a .6 m$ & $9 \mathrm{~m}$ \\
\hline Falou & 2 anos & $9 \mathrm{~m}$ & 2 anos & $9 \mathrm{~m}$ & $2 a .6 m$ & --- & $2 a .7 m$ & $\begin{array}{c}+ \text { de } 1 \\
\text { ano }\end{array}$ & --- & $\begin{array}{c}+ \text { de } 2 \\
\text { anos }\end{array}$ \\
\hline
\end{tabular}

Nota. A idade cronológica refere-se àquela do início da coleta de dados. Os traços (---) indicam que as mães não se lembravam das idades em que suas crianças sustentaram a cabeça e falaram. Os números 1, 2, 3, 4 e 5 identificam as crianças em cada tipo de família. 
Com relação às atividades de vida diária, tais como toalete, banho, alimentação e sono, as crianças DT foram descritas pelas mães como apresentando uma independência maior que as crianças SD. Estas estavam 'Desenvolvendo' as habilidades para se tornarem independentes em relação à alimentação $(n=4)$, ao sono $(n=4)$ e aos hábitos de toalete $(n=3)$, enquanto a maioria das crianças DT era 'Independente' nestes aspectos (alimentação $n=3$; sono $n=4$; hábitos de toalete $n=3$ ). Quanto ao banho, tanto as crianças SD $(n=5)$, quanto a maioria das crianças DT $(n=3)$ era 'Dependente', isto é, elas necessitavam da ajuda de um adulto para a realização desta atividade.

No que se refere às características comportamentais das crianças, a maioria dos genitores percebeu sua criança como sendo 'Alegre'. As crianças SD foram descritas também como sendo 'Irritadas' e 'Calmas', além de 'Birrentas', enquanto as crianças DT como sendo 'Sensíveis' e 'Agitadas'. As crianças, tanto as SD como as DT, quando contrariadas, costumavam apresentar dois tipos de comportamentos: 'Nervosismo' $(n=5)$ e 'Emburramento' $(n=5)$. Nestes momentos, os genitores relataram reagir com 'Punição verbal' ( $n=5$ pais; $n=3$ mães), 'Ação Reforçadora' ( $n=2$ pais; $n=2$ mães), 'Indiferença' ( $n=2$ pais; $n=$ 2 mães), 'Punição mista' ( $n=1$ mãe), 'Punição física' ( $n=1$ mãe) ou 'Não punição' ( $n=1$ pai; $n=1$ mãe).

No período de dois anos, foram mais freqüentes as mudanças ocorridas com as crianças com desenvolvimento típico do que com as crianças com síndrome de Down, segundo o relato das mães. De modo geral, todas as crianças tiveram melhorias, principalmente, nos aspectos do desenvolvimento neuropsicomotor, de linguagem e de sociabilidade. Quanto à escolarização, três crianças DT foram consideradas como tendo adquirido os conhecimentos básicos de sua série. O Quadro 1 apresenta as mudanças no desenvolvimento de cada uma das crianças, tal qual percebido pelas mães.

Quadro 1. Mudanças ocorridas com as crianças durante a coleta de dados

\begin{tabular}{|c|c|c|c|c|c|c|c|c|c|c|}
\hline \multirow{2}{*}{$\begin{array}{l}\text { Aspectos do desenvolvimento } \\
\text { Neuropsicomotor e linguagem }\end{array}$} & \multicolumn{10}{|c|}{ Crianças } \\
\hline & SD1 & DT1 & SD2 & DT2 & SD3 & DT3 & SD4 & DT4 & SD5 & DT5 \\
\hline $\begin{array}{l}\text { Aquisição do controle esfincteriano } \\
\text { Começou a andar } \\
\text {. Melhoria no desenvolvimento global } \\
\text {. Desenvolvimento do raciocínio lógico } \\
\text {. Maior rapidez de raciocínio } \\
\text {. Melhoria da coordenação motora } \\
\text {. Melhoria da linguagem }\end{array}$ & 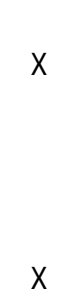 & $X$ & & $X$ & $x$ & $\begin{array}{l}x \\
x\end{array}$ & $\begin{array}{l}x \\
x\end{array}$ & $X$ & $X$ & $X$ \\
\hline $\begin{array}{l}\text { Escolarização } \\
\text {. Aquisição de conhecimentos básicos } \\
\text {. Início da pré-escola no ensino regular } \\
\text { Término do período formal de frequêencia à } \\
\text { Estimulação Precoce (4 anos de freqüência) } \\
\text { Abandono de freqüência ao atendimento da } \\
\text { Estimulação Precoce }\end{array}$ & & $x$ & & $\begin{array}{l}X \\
X\end{array}$ & $X$ & & $X$ & $X$ & $\begin{array}{l}x \\
x\end{array}$ & \\
\hline $\begin{array}{l}\text { Sociabilidade } \\
\text { Melhoria na sociabilização }\end{array}$ & & $X$ & $X$ & $X$ & & $X$ & $X$ & & $X$ & $x$ \\
\hline $\begin{array}{l}\text { Comportamento } \\
\text {. Melhoria do comportamento na escola }\end{array}$ & & & & & & & & & & $X$ \\
\hline $\begin{array}{l}\text { Aspectos da saúde } \\
\text { Ocorrência de doenças } \\
\text { Ocorrência de internações }\end{array}$ & $X$ & $\begin{array}{l}X \\
X\end{array}$ & & & $X$ & $x$ & $x$ & & $x$ & $\begin{array}{l}X \\
X\end{array}$ \\
\hline
\end{tabular}

Nota. Os números 1, 2, 3, 4 e 5 identificam as crianças em cada tipo de família.

Com relação aos relacionamentos sociais, a maioria das mães das crianças $\mathrm{SD}(n=4)$ e DT $(n=4)$ relatou não ter havido alterações nas relações da criança com elas e com o pai. As relações entre os irmãos foram as que mais alteraram ao longo de dois anos, caracterizando-se por uma 'maior proximidade entre os irmãos'. 


\section{O modo de vida das famílias}

A rotina das famílias durante a semana era semelhante. Em geral, o pai ou ambos os genitores trabalhavam fora de casa, em período integral, só retornando à noite. Todas as crianças SD freqüentavam o Programa de Estimulação Precoce, em período parcial, e uma delas freqüentava o ensino regular, no outro período. Três crianças DT freqüentavam a pré-escola ou a creche, enquanto duas não haviam iniciado o processo de escolarização formal. A maioria dos irmãos $(n=$ 14) freqüentava a escola; os outros ficavam em casa $(n=3)$. As atividades de lazer eram similares para ambos os tipos de famílias $(n=8)$, sendo estas realizadas mais freqüentemente nos finais de semana, tendo, em geral, a participação do pai, da mãe e dos filhos $(n=6)$ ou somente da mãe e dos filhos $(n=4)$.

Com relação aos cuidados dispensados às crianças, 0 pai e a mãe compartilhavam as seguintes tarefas: dar comida/banho $(n=3)$, levar/trazer da escola $(n=2) \mathrm{e}$ colocar para dormir $(n=3)$. No entanto, em duas famílias, a mãe assumia todas as tarefas e, em três, as famílias contavam com a ajuda de empregadas/babá. Quando os genitores estavam ausentes, a criança costumava ficar com a empregada $(n=2)$, com a babá $(n=1)$, com irmãos mais velhos $(n=1)$ ou, ainda, permanecia na creche o dia todo $(n=1)$. Nas demais famílias $(n=5)$, as crianças ficavam com a mãe. Ao término da coleta de dados, houve uma mudança na divisão de responsabilidades domésticas, tendo aumentado o número de crianças cuidadas por empregadas $(n=3)$ e por irmãos mais velhos $(n=2)$, havendo uma conseqüente diminuição de famílias cujas crianças costumavam ficar com a mãe o dia todo $(n=3)$.

Outras mudanças foram relatadas pelas famílias durante o período de dois anos de coleta de coleta de dados. As freqüências das alterações ocorridas na rotina das famílias podem ser verificadas na Figura 1.

\section{$\square$ Síndrome de Down $\square$ Desenvolvimento típico}

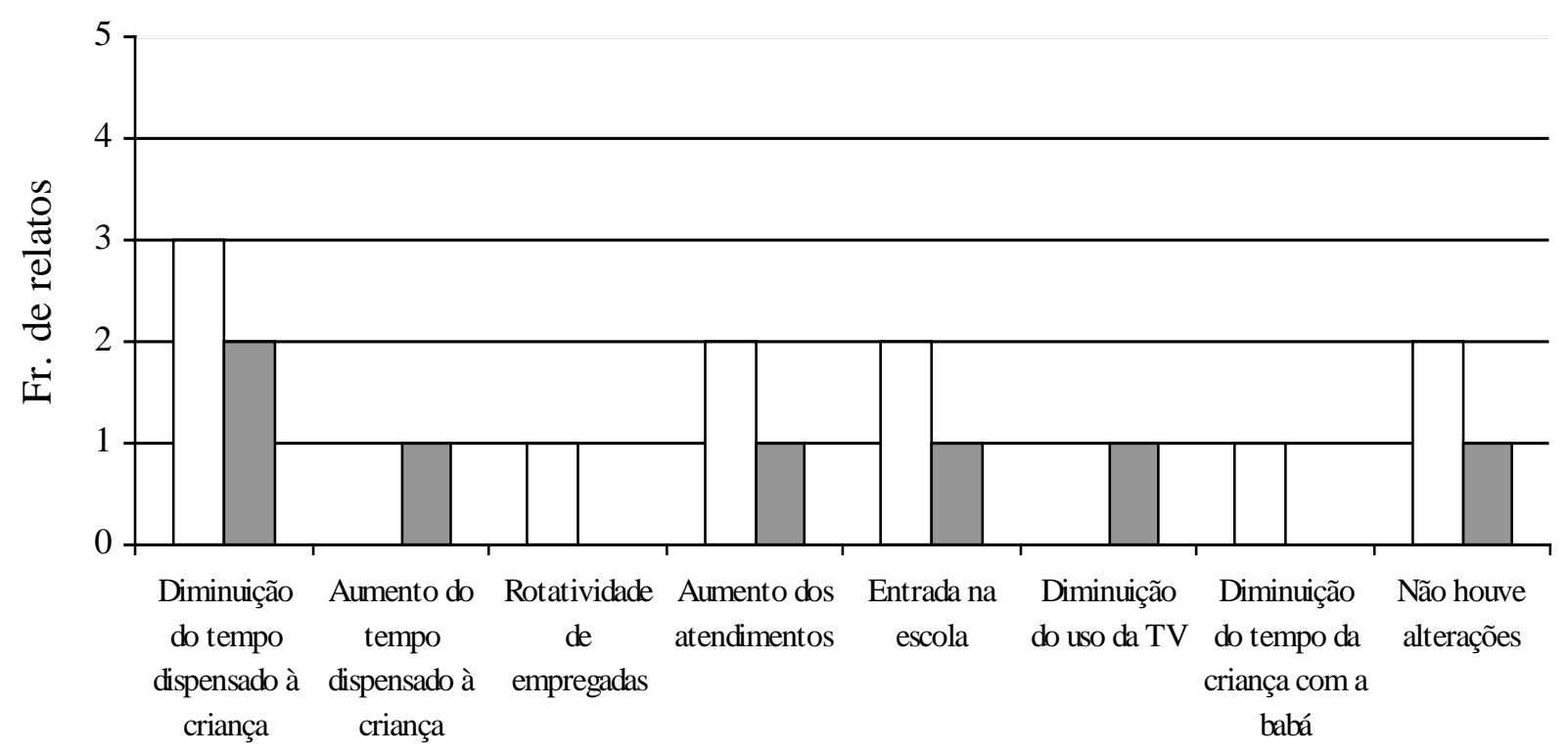

Figura 1. Alterações na rotina das famílias no decorrer de dois anos.

Os fatos novos mais freqüentemente relatados foram: 'início em novo emprego' $(n=4)$, 'morte/acidente com parentes próximos' $(n=4)$ e 'reforma da casa' $(n=3)$. Nas famílias de crianças SD houve um 'aumento das despesas com os filhos' $(n=1)$ e, nas famílias de crianças DT, foram mencionadas mudanças no trabalho, isto é, 'mudança de cargo na empresa' $(n=2)$. 


\section{O nível de estresse parental nas famílias de crianças com e sem Síndrome de Down}

Quanto mais elevado o escore, maior o nível de estresse. Como este instrumento (PSI) ainda não foi validado no Brasil, não se tem um valor mínimo indi- cativo de estresse para a nossa população; sendo assim, foram utilizados os escores brutos para efeitos de comparação entre as famílias. A Figura 2 apresenta os escores brutos do nível de estresse total de cada genitor, segundo os tipos de família.

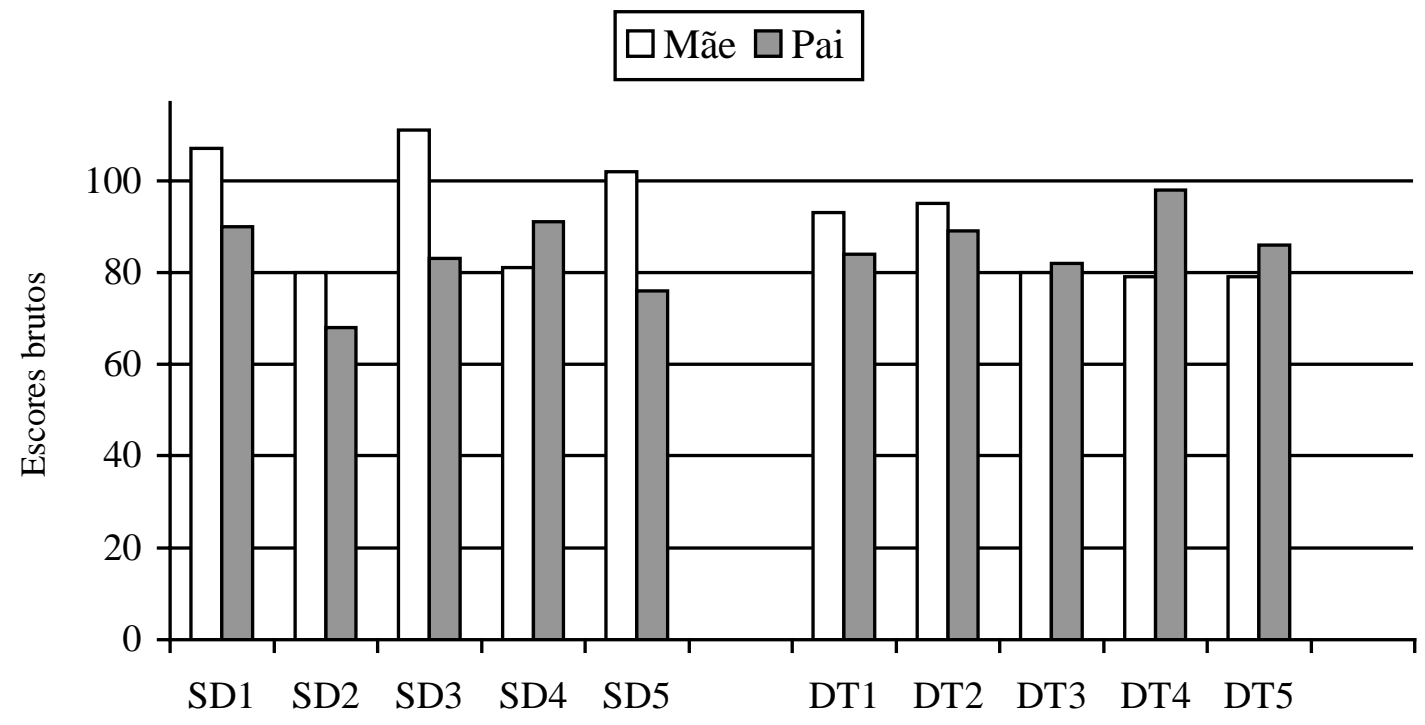

Figura 2. Escores brutos do nível de estresse parental.

É interessante destacar que a maioria das mães de crianças $\mathrm{SD}(n=3)$ obteve um escore maior $(\mathrm{M}=$ $96,2)$ que as mães de crianças DT $(M=85,2)$, enquanto com os pais ocorreu o inverso, isto é, os pais de crianças DT percebiam mais estresse decorrente de suas relações com os filhos $(\mathrm{M}=87,8)$ do que os pais de crianças SD $(\mathrm{M}=81,6)$. Comparando o grupo de mães $(n=10, \mathbf{M}=90,7)$ com o grupo de pais $(n=10$, $M=84,7)$, verifica-se que as mães apresentam escores mais altos que estes últimos, indicando que se sentem e se percebem mais estressadas que os pais. O estresse parental depende, em parte, de como os genitores percebem a si próprios em seus papéis maternos e paternos.

Como os genitores dessas famílias se sentem em seus papéis de pai e mãe? Esta questão pode ser respondida através da análise pormenorizada da escala 'sofrimento parental'. Em nosso estudo, tanto as mães $(n=10, \mathrm{M}=29,9)$ quanto os pais $(n=10, \mathrm{M}=27,2)$ obtiveram índices abaixo do mínimo, sugerindo que estes genitores não se sentem angustiados no desempenho de seus papéis parentais. Nesta sub-escala, as médias obtidas pelo grupo de mães de crianças SD e
DT (SD: $M=30,6$; DT: $M=29,2$ ) e aquelas obtidas pelo grupo de pais de crianças SD e DT (SD: $\mathrm{M}=$ 26,6; DT: $M=27,8$ ) foram similares.

$\mathrm{Na}$ segunda sub-escala, 'interações disfuncionais entre genitor-criança', as médias de pais e mães estão abaixo do escore bruto mínimo (mães: $\mathrm{M}=26,7$; pais: $M=25,2)$, sugerindo que ambos não percebem dificuldades na relação parental. Com relação às diferenças entre os tipos de família, as mães de crianças SD obtiveram uma média de 27,6, enquanto as mães de crianças DT obtiveram uma média de 24,2 . Já os pais de crianças SD apresentaram uma média de 23,6 e os de crianças DT de 26,8 .

A análise da sub-escala 'criança difícil' indica que a maioria dos genitores não percebe sua criança como 'difícil' de manejar, uma vez que a média para o grupo de mães foi 34,1 e para o de pais 32,3. A média das mães de crianças SD foi mais alta $(M=36,4)$ que das mães de crianças DT $(M=31,8)$, sugerindo que as primeiras percebem um pouco mais de dificuldades com suas crianças relacionadas ao temperamento das mesmas. A média para os pais de ambos os grupos também foi similar (SD: $\mathrm{M}=31,4$; $\mathrm{DT}: \mathrm{M}=33,2$ ). 
O estresse parental em famílias de crianças SD está também relacionado às reações e sentimentos dos genitores frente ao diagnóstico recebido por ocasião do nascimento da criança SD. Na maioria dessas famílias $(n=3)$, as mães, ainda no hospital, receberam a notícia sobre a suspeita de que a criança tinha síndrome de Down; uma delas na própria sala de parto. Nas outras duas famílias, foram os pais que receberam a notícia sobre a suspeita, também no hospital.
A confirmação do diagnóstico foi recebida somente pelas mães em duas das famílias e, em três delas, o pai e a mãe receberam, juntos, essa notícia. A maioria das mães $(n=3)$ não soube especificar em que época teve a confirmação do diagnóstico e duas delas relataram que a confirmação foi obtida no intervalo de uma semana a um mês e meio após o nascimento da criança. A Tabela 2 apresenta os tipos de reação e sentimentos relatados pelos genitores diante da suspeita e da confirmação do diagnóstico.

Tabela 2. Tipos de reação e sentimento da mãe e do pai diante da suspeita e da confirmação do diagnóstico de sua criança

\begin{tabular}{l|cc|cc|cc}
\hline \multirow{2}{*}{ Categoria } & \multicolumn{2}{|c|}{ À suspeita } & \multicolumn{2}{|c|}{ À confirmação } & \multicolumn{3}{c}{ TOTAL } \\
& Mãe & Pai & Mãe & Pai & Mãe & Pai \\
& Fr. & Fr. & Fr. & Fr. & Fr. & Fr. \\
\hline Reações & & & & & & \\
. Aceitação & 1 & - & 2 & 3 & 3 & 3 \\
Negação & 1 & 1 & 2 & 1 & 3 & 2 \\
. Rejeição & 1 & 2 & 1 & - & 2 & 2 \\
Não relatado & 2 & 2 & - & 1 & 2 & 3 \\
Sentimentos & & & & & & \\
. Incerteza & 3 & 1 & 2 & - & 5 & 1 \\
. Impotência & - & 1 & - & 1 & - & 2 \\
. Revolta & 1 & - & 3 & 1 & 4 & 1 \\
Sofrimento & - & 1 & 3 & 1 & 3 & 2 \\
. Choque & - & - & - & 2 & - & 2 \\
Não relatado & 1 & 2 & 2 & 2 & 3 & 4 \\
\hline
\end{tabular}

Nota. Foram relatados mais de um tipo de sentimento pelo mesmo genitor.

Após o diagnóstico, a principal decisão tomada por todas as famílias foi a de 'Procurar ajuda'. O tipo de ajuda mais procurada foi a 'Especializada', tanto de 'Profissionais individuais' $(n=2)$ como de 'Centros' especializados $(n=3)$ e, neste caso, centros de ensino especial que ofereciam um Programa de Estimulação Precoce. A iniciativa de procurar ajuda, na maioria das famílias $(n=3)$, foi tomada em conjunto pelo pai e pela mãe; apenas em duas delas a mãe tomou a iniciativa sozinha.

\section{DISCUSSÃO}

O momento da notícia do diagnóstico parece ser o mais sofrido para os pais, como mostra a literatura (Brunhara \& Petean, 1999; Casarin, 1999; Colnago \& Biasoli-Alves, 2003; Pereira-Silva \& Dessen, 2003; Rodrigues, Lopes, Zuliani, Marques \& Combinato, 2003). É neste momento que a família pode promover a construção de fantasias a respeito da criança e sua deficiência e, por isso, merece uma atenção mais espe- cial dos profissionais envolvidos (Brunhara \& Petean, 1999; Pereira-Silva \& Dessen, 2002; Petean, 1995; Rodrigues \& cols., 2003). Segundo Araújo (2004), é importante que se estabeleça uma comunicação efetiva entre a família e o profissional, o que acarreta benefícios para todos: família, criança com deficiência, profissional e comunidade.

Quando os genitores recebem a notícia de suspeita ou a confirmação do diagnóstico de síndrome de Down, as reações e os sentimentos são variados (Regen \& cols., 1993) e diferem entre pais e mães (Glat \& Duque, 2003; Minnes, 1998), o que é corroborado pelos dados deste estudo. Considerando que o momento do diagnóstico representa um ponto crítico para as interações familiares futuras, podendo gerar um desequilíbrio no funcionamento da família, é de fundamental importância que os profissionais sejam melhor preparados para lidar com os pais, conforme vem sendo ressaltado pela literatura (Brunhara \& Petean, 1999). 
De acordo com o relato dos genitores deste estudo, todas as crianças com SD eram dependentes para se alimentar, para dormir e, também, nos hábitos de toalete. Por outro lado, a maioria do grupo com DT era independente em tais aspectos. Contudo, os genitores das crianças com síndrome de Down percebiam que seus filhos estavam desenvolvendo as habilidades necessárias para a aquisição da independência, embora de forma gradual e diferenciada das demais crianças, o que é coerente com a lentidão no desenvolvimento de crianças com SD (Saad, 2003). Esta percepção reflete uma postura positiva dos pais no sentido de que, embora as crianças SD apresentem dificuldades, não se mostram impossibilitadas de se desenvolverem, conforme valores e crenças que vigoraram por décadas e décadas. Por longo tempo, estas crianças eram tidas como incapazes e, portanto, impedidas de participar ativamente na sociedade (Glat, 1995; Pereira-Silva \& Dessen, 2001; Pessoti, 1984).

Segundo o relato dos genitores, as crianças SD não apresentavam mais problemas de comportamento que as crianças DT, o que é inconsistente com a maioria dos estudos relatados pela literatura (por exemplo, Cuskelly \& Dadds, 1992; Floyd \& Zmich, 1991; Leary \& Verth, 1995; Turnbull \& Ruef, 1996). Um exemplo a ser destacado é a pesquisa de Floyd, Harter e Costigan (2004) com 162 famílias de crianças com DM, com doenças crônicas e sem DM ou doenças crônicas, as quais foram observadas em situações de resolução de problemas. Os resultados mostraram que os problemas de comportamento da criança estavam relacionados às interações negativas entre os genitores e sua criança. Neste mesmo sentido, concluiu-se que níveis altos de coesão familiar e independência, bem como baixos níveis de falta de entrosamento e de compromisso estavam associados a poucas trocas negativas entre os genitores e suas crianças. No contexto brasileiro, Pereira-Silva e Dessen (2006) mostram que as interações genitores-criança SD são caracterizadas, em sua maioria, por sincronia, amistosidade e liderança dos genitores. Os dados da literatura sugerem, portanto, a necessidade de se investigar mais profundamente as relações entre os problemas de comportamento e a qualidade das interações familiares, sobretudo no Brasil, que carece de estudos a respeito (Dessen \& Pereira-Silva, 2000).

As mudanças ocorridas nas famílias deste estudo, ao longo dos dois anos de coleta de dados, podem ser interpretadas à luz da abordagem bioecológica (Bronfenbrenner, 1979/1996, 1999). Por exemplo, a diminuição do tempo dispensado pelos genitores à criança ocorrida na rotina das famílias, é, em parte, decorrente do aumento da crise econômica enfrentada no país (questões relativas ao macrossistema), exigindo que algumas mães passassem a trabalhar fora de casa. Assim, início em novo emprego, mudança de cargo na empresa e diminuição do uso da TV sugerem a influência do exossistema no microssistema familiar. As relações mantidas pelos genitores com a escola (mesossistema), por sua vez, tiveram impacto no desenvolvimento das crianças. É curioso que todas as modificações relatadas não foram associadas, pela maioria dos genitores, às alterações nas interações entre eles e sua criança, mas somente entre esta e os irmãos. Por exemplo, há consenso na literatura que a diminuição do tempo dispensado à criança é uma variável que pode interferir no modo como os genitores se relacionam com os filhos (Sinha, 1995).

Conforme mencionado anteriormente, o estresse parental interfere nas relações entre os genitores e sua criança e, conseqüentemente, no desenvolvimento infantil. A análise dos escores brutos da Escala de Estresse Parental-PSI obtidos em nosso estudo mostra que o nível de estresse foi ligeiramente superior entre as mães de crianças com SD do que entre as mães de crianças com DT, o que é consistente com a literatura (Dyson, 1997; Hornby, 1995; Lamb \& Billings, 1997; Shapiro \& cols., 1998; Sloper \& cols., 1991). É perfeitamente compreensível que tais mães se percebessem mais estressadas, já que elas eram as principais responsáveis pelos cuidados de sua criança. Ser a principal responsável pelos cuidados dos filhos acarreta sobrecarga psicológica, desencadeando o estresse vivenciado pela maioria dos genitores de crianças com deficiência (Ali \& cols., 1994; Shapiro \& cols., 1998). Apesar de os resultados do PSI terem indicado maior nível de estresse entre as mães de crianças com SD, elas não relataram, nas entrevistas, estar vivenciando uma situação estressante.

Em contrapartida, os pais de crianças com DT obtiveram escores ligeiramente mais altos que os pais de crianças com SD, tanto na totalização do estresse como em algumas sub-escalas, o que não era esperado. A literatura ressalta que pais de crianças com SD apresentam um estresse mais elevado que pais de crianças com DT, em parte devido à sobrecarga financeira e de cuidados dispensados à criança (Ali \& cols., 1994; Rodrigue \& cols., 1992). Isto nos faz refletir sobre a importância da influência de fatores culturais sobre os níveis de estresse de pais brasileiros. Em 
nossa cultura, apesar de a responsabilidade pela manutenção e cuidados da casa e dos filhos ser atribuída à mulher (Dessen \& Torres, 2002; Romanelli, 1998, 2000), há outros fatores inter-relacionados que precisam ser investigados para que se possa compreender o estresse parental em famílias de crianças com SD.

A análise da sub-escala 'sofrimento parental' indica que os genitores de ambos os tipos de famílias parecem vivenciar níveis semelhantes de dificuldades de relacionamento com seus filhos. Contudo, nas subescalas 'disfunções na interação genitor-criança' e 'criança difícil', as mães de crianças com SD obtiveram escores mais altos que as mães de crianças com DT, o que é compreensível, na medida em que a responsabilidade e a sobrecarga de cuidados com a criança com SD são também maiores (Ali \& cols., 1994; Shapiro \& cols., 1998). Já, os pais de crianças com DT obtiveram escores mais elevados que os pais de crianças com SD nestas sub-escalas, sugerindo que os primeiros apresentam mais dificuldades de relacionamento com seus filhos. Tais dificuldades podem estar associadas ao nível de expectativas dos pais em relação à sua criança. Embora os pais de ambos os grupos tenham relatado expectativas similares nas entrevistas, os pais de crianças com SD, na realidade, acreditam que não podem esperar muito de suas crianças. Outra fonte de explicação refere-se às influências indiretas nas relações (Bronfenbrenner, 1979/1996, 1999), em que os pais de crianças com DT podem estar vivenciando mais estresse em decorrência das influências do ambiente do trabalho e também de suas relações maritais (Erel \& Burman, 1995; Stoneman \& Gavidia-Payne, 2006). Tais dimensões não foram investigadas neste estudo.

Em suma, os dados deste estudo indicam que não há diferenças acentuadas no funcionamento das famílias de crianças com SD e com DT, no que tange ao estresse parental e aos modos de vida familiar. As diferenças encontradas podem ser explicadas pelas diferenças que comumente ocorrem nos padrões de comunicação de uma família para outra, em uma dada cultura, em decorrência de fatores sociais, educacionais etc. (Torres \& Dessen, 2006), independentemente de haver criança com algum tipo de deficiência.

\section{REFERÊNCIAS}

Abidin, R. R. (1995). Parenting stress index. Odessa, FL: Psychological Assessement Resources.

Ali, M. R., Al-Shatti, A., Khaleque, A., Rahman, A., Ali, S. M. \& Ahmed, R. U. (1994). Parents of mentally retarded children: personality characteristics and psychological problems. Social Behavior and Personality, 22, 41-52.

Araújo, E. A. C. (2004). Parceria família-profissional em educação especial: promovendo habilidades de comunicação efetiva. Em E. G. Mendes, M. A. Almeida \& L. C. A. Williams (Orgs.), Temas em educação especial: avanços recentes (pp. 175-178). São Carlos: EdUFSCar.

Bigras, M., Venet, M. \& Normandeau, S. (2002, agosto). The relation between cognitive and socioaffective predictors of academic performance at the end of first grade. Trabalho apresentado no 17th Meeting of the International Society for the Study of Behavioral Development, Ottawa, Canadá.

Bristol, M. M., Gallagher, J. J. \& Schopler, E. (1988). Mothers and fathers of young developmentally disabled and nondisabled boys: adaptation and spousal support. Developmental Psychology, 24, 441-451.

Bronfenbrenner, U. (1996). A ecologia do desenvolvimento humano: experimentos naturais e planejados. Porto Alegre: Artes Médicas. (Trabalho originalmente publicado em 1979)

Bronfenbrenner, U. (1999). Environments in developmental perspective: theoretical and operational models. Em S. L. Friedman \& T. D. Wachs (Orgs.), Measuring environment across the life span: Emerging methods and concepts (pp. 3-28). Washington, DC: American Psychological Association.

Brunhara, F. \& Petean, E. B. L. (1999). Mães e filhos especiais: reações, sentimentos e explicações à deficiência da criança. Cadernos de Psicologia e Educação Paidéia, 9, 31-40.

Carr, J. (1988). Six weeks to twenty-one years old: a longitudinal study of children with Down's syndrome and their families. Journal of Child Psychology and Psychiatry, 29, 407-431.

Casarin, S. (1999). Aspectos psicológicos na síndrome de Down. Em J. S. Schwartzman (Org.), Síndrome de Down (pp. 263285). São Paulo: Mackenzie.

Colnago, N. A. S. \& Biasoli-Alves, Z. (2003). Necessidades de famílias de bebês com síndrome de Down - SD: subsídios para uma proposta de intervenção. Em M.C. Marquezine, M. A. Almeida, S. Omote \& E. D. O. Tanaka (Orgs.), O papel da família junto ao portador de necessidades especiais (pp.1-14 ). Londrina: EDUEL.

Cuskelly, M. \& Dadds, M. (1992). Behavioural problems in children with Down's syndrome and their siblings. Journal of Child Psychology and Psychiatry, 33, 749-761.

Dessen, M. A. \& Pereira-Silva, N. L. (2000). Deficiência mental e família: uma análise da produção científica. Cadernos de Psicologia e Educação Paidéia, 10, 12-23.

Dessen, M. A. \& Torres, C. (2002). Family and socialization factors in Brazil: an overview. Em W. J. Lenner, D. L. Dinnel, S. A. Hayes \& D. N. Sattler (Orgs.), OnLine Readings in Psychology and Culture (Unit 13, Chapter 2). Bellingham, Washington, USA: Western Washington University, Center for Cross-Cultural Research.. Obtido em 02/12/07 do World Wide Web:htttp://www.wwu.edu/ culture.

Dyson, L. L. (1997). Fathers and mothers of school-age children with developmental disabilities: parental stress, family functioning, and social support. American Journal on Mental Retardation, 102, 267-279.

Interação em Psicologia, Curitiba, jul./dez. 2006, (10)2, p. 183-194 
Erel, O. \& Burman, B. (1995). Interrelatedness of marital relations and parent-child relations: a meta-analytic review. Psychological Bulletin, 118, 108-132.

Floyd, F. J., Harter, K. S. M. \& Costigan, C. L. (2004). Family problem-solving with children who have mental retardation. American Journal on Mental Retardation, 109, 507-524.

Floyd, F. J. \& Zmich, D. E. (1991). Marriage and the parenting partnership: perceptions and interactions of parents with mentally retarded and typically developing children. Child Development, 62, 1434-1448.

Glat, R. (1995). Integração dos portadores de deficiências: uma questão psicossocial. Temas em Psicologia, 2, 89-94.

Glat, R. \& Duque, M. A. (2003). Convivendo com filhos especiais: o olhar paterno. Rio de Janeiro: Viveiros de Castro Editora Ltda.

Glidden, L. M. \& Floyd, F. J. (1997). Disaggregating parental depression and family stress in assessing families of children with developmental disabilities: a multisample analysis. American Journal on Mental Retardation, 102, 250-266.

Hastings, R. P., Daley, D. \& Burns, C. (2006). Maternal distress and expressed emotion: cross-sectional and longitudinal relationships with behavior problems of children with intellectual disabilities. American Journal on Mental Retardation, 11, 4861 .

Hornby, G. (1995). Effects on fathers of children with Down syndrome. Journal of Child and Family Studies, 4, 239-255.

Krauss, M. W. \& Seltzer, M. M. (1998). Life course perspectives in mental retardation research: the case of family caregiving. Em J. A. Burack, R. M. Hodapp \& E. Zigler (Orgs.), Handbook of mental retardation and development (pp. 504-520). Cambridge: Cambridge University Press.

Lamb, M. E. \& Billings, L. A. L. (1997). Fathers of children with special needs. Em M.E. Lamb (Org.), The role of the father in child development (pp. 179-190). New York: Wiley.

Leary, P. M. \& Verth, F. (1995). The effect of a mentally retarded child on family functioning in a third world community. Early Child Development and Care, 109, 83-88.

Minnes, P. (1998). Mental retardation: the impact upon the family. Em J. A. Burack, R.M. Hodapp \& E. Zigler (Orgs.), Handbook of mental retardation and development (pp. 693-712). Cambridge: Cambridge University Press.

Negrin, N. S. \& Cristante, F. (1996). Resources and stress in parents with a mentally retarded child: a quantitative approach. Em M. Cusinato (Org.), Research on family: resources and needs across the world (pp. 493-506). Milão: LED - Edicioni Universitarie.

Pereira-Silva, N. L. \& Dessen, M. A. (2001). Deficiência mental e família: implicações para o desenvolvimento da criança. Psicologia: Teoria e Pesquisa, 17, 133-141.

Pereira-Silva, N. L. \& Dessen, M. A. (2002). Síndrome de Down: etiologia, caracterização e impacto na família. Interação em Psicologia, 6, 167-176.

Pereira-Silva, N. L. \& Dessen, M. A. (2003). Crianças préescolares com síndrome de Down e suas interações familiares. Psicologia: Reflexão e Crítica, 16, 503-514.

Pereira-Silva, N. L. \& Dessen, M. A. (2004). O que significa ter uma criança deficiente mental na família? Educar em Revista, 23, 161-183.
Pereira-Silva, N. L. \& Dessen, M. A (2006). Padrões de interação genitores-crianças com e sem síndrome de Down. Psicologia: Reflexão e Crítica, 19, 283-291.

Pessoti, I. (1984). Deficiência mental: da superstição à ciência. São Paulo: Editora da Universidade de São Paulo.

Petean, E. B. L. (1995). Avaliação qualitativa dos aspectos psicológicos do aconselhamento genético através do estudo prospectivo do atendimento das famílias. Tese de Doutorado, Faculdade de Ciências Médicas da Unicamp, Campinas.

Regen, M., Ardore, M. \& Hoffmann, V. M. (1993). Mães e filhos especiais: relato de experiência com grupos de mães de crianças com deficiência. Brasília: Corde.

Rodrigue, J. R., Morgan, S. B. \& Geffken, G. R. (1992). Psychosocial adaptation of fathers of children with autism, Down syndrome and normal development. Journal of Autism and Development Disorders, 22, 249-263.

Rodrigues, O. M. P. R., Lopes, A. A., Zuliani, G., Marques, L. C. \& Combinato, D. S. (2003). Pais de bebês com anomalias craniofaciais: análise das reações após o nascimento e atualmente. Em M.C. Marquezine, M. A. Almeida, S. Omote \& E. D. O. Tanaka (Orgs.), O papel da família junto ao portador de necessidades especiais (pp. 15-22). Londrina: EDUEL.

Romanelli, G. (1998). O relacionamento entre pais e filhos em famílias de camadas médias. Cadernos de Psicologia e Educação Paidéia, 8, 123-136.

Romanelli, G. (2000). Autoridade e poder na família. Em M. C. B. Carvalho (Org.), A família contemporânea em debate ( $3^{\mathrm{a}}$ ed., pp. 73-88). São Paulo: EDUC.

Saad, S. N. (2003). Preparando o caminho da inclusão: dissolvendo mitos e preconceitos em relação à pessoa com síndrome de Down. São Paulo: Vetor.

Shapiro, J., Blacher, J. \& Lopez, S. R. (1998). Maternal reactions to children with mental retardation. Em J.A. Burack, R. M. Hodapp \& E. Zigler (Orgs.), Handbook of mental retardation and development (pp. 606-636). Cambridge: Cambridge University Press.

Singer, G. H. S. (2006). Meta-analysis of comparative studies of depression in mothers of children with and without developmental disabilities. American Journal on Mental Retardation, $111,155-169$.

Sinha, S. R. (1995). Childrearing practices relevant for the growth of dependency and competence in children. Em J. Valsiner (Org.), Child development within culturally structured environments (pp. 105-135). Norwood, N. J: Ablex Publishing Corporation.

Slonims, V. \& McConachie, H. (2006). Analysis of mother-infant interaction in infants with Down syndrome and typically developing infants. American Journal on Mental Retardation, 111, 273-289.

Sloper, P., Knussen, C., Turner, S. \& Cunningham, C. (1991). Factors related to stress and satisfaction with life in families of children with Down's syndrome. Journal of Child Psychology and Psychiatry, 32, 655-676.

Stoneman, Z. \& Gavidia-Payne, S. (2006). Marital adjustment in families of young children with disabilities: associations with daily hassles and problem-focused coping. American Journal on Mental Retardation, 111, 1-14. 
Torres, C. \& Dessen, M. A. (2006). The Brazilian 'jeitinho': Brazil's sub-cultures, its diversity of social contexts, and family structures. Em J. Georgas, J. W. Berry, F. J. R. van de Vijver, C. Kagitcibasi \& Y. H. Poortinga (Orgs.), Family across cultures: a 30 nation psychological study (pp. 259-266). Cambridge, UK: Cambridge University Press.

Recebido: $14 / 10 / 2006$

Turnbull, A. P. \& Ruef, M. (1996). Family perspectives on proRevisado: 15/12//2006 blem behavior. Mental Retardation, 34, 280-293.

Aceito: $26 / 12 / 2006$

\section{Notas:}

${ }^{1}$ Este artigo foi escrito com base nos resultados da Tese de Doutorado apresentada ao Instituto de Psicologia da Universidade de Brasília pela primeira autora, sob orientação da segunda. As autoras agradecem o apoio recebido do CNPq.

${ }^{2}$ Crianças que apresentam características típicas do ponto de vista do desenvolvimento humano, ou seja, não são diagnosticadas, rotuladas ou classificadas por profissionais da área de saúde como apresentando algum tipo de anomalia ou doença, seja física, mental ou psicológica.

\section{Sobre as autoras:}

Nara Liana Pereira-Silva: É psicóloga, com Doutorado em Psicologia pela Universidade de Brasília. Desenvolve pesquisas sobre relações familiares de crianças com deficiência mental no Laboratório de Desenvolvimento Familiar - IP/UnB. É professora do curso de Psicologia no Instituto de Educação Superior de Brasília - IESB e psicóloga da Secretaria de Estado de Educação do Distrito Federal. Endereço eletrônico: liana@unb.br

Maria Auxiliadora Dessen: Doutora em Psicologia pela Universidade de São Paulo, com pós-doutoramento pela Universidade de Lancaster, Inglaterra, e pelo Instituto Max Planck para o Desenvolvimento Humano-MPI, Berlim, Alemanha. É professora do Instituto de Psicologia da Universidade de Brasília-UnB, coordenadora do Laboratório de Desenvolvimento Familiar (IP/UnB), coordenadora do Programa de Pós-Graduação em Processos de Desenvolvimento Humano e Saúde (IP/PED/UnB) e pesquisadora do CNPq. Endereço eletrônico: dessen@unb.br

Endereço para correspondência: Universidade de Brasília-UnB; Instituto de Psicologia/PED; Laboratório de Desenvolvimento Familiar -Campus Universitário Darcy Ribeiro - 70910-900 Brasília/DF 